\title{
Real-time turbulence profiling with a pair of laser guide star Shack-Hartmann wavefront sensors for wide-field adaptive optics systems on large to extremely large telescopes
}

\author{
L. Gilles* and B. L. Ellerbroek \\ Thirty Meter Telescope Observatory Corporation, 1200 E. California Boulevard, Mail Code 102-8, Pasadena, \\ California 91125, USA \\ *Corresponding author: lgilles@caltech.edu
}

Received April 8, 2010; revised July 30, 2010; accepted August 6, 2010;

posted August 6, 2010 (Doc. ID 126706); published August 31, 2010

\begin{abstract}
Real-time turbulence profiling is necessary to tune tomographic wavefront reconstruction algorithms for widefield adaptive optics (AO) systems on large to extremely large telescopes, and to perform a variety of image post-processing tasks involving point-spread function reconstruction. This paper describes a computationally efficient and accurate numerical technique inspired by the slope detection and ranging (SLODAR) method to perform this task in real time from properly selected Shack-Hartmann wavefront sensor measurements accumulated over a few hundred frames from a pair of laser guide stars, thus eliminating the need for an additional instrument. The algorithm is introduced, followed by a theoretical influence function analysis illustrating its impulse response to high-resolution turbulence profiles. Finally, its performance is assessed in the context of the Thirty Meter Telescope multi-conjugate adaptive optics system via end-to-end wave optics Monte Carlo simulations. (c) 2010 Optical Society of America

OCIS codes: $010.1080,010.7350,010.1330$
\end{abstract}

\section{INTRODUCTION}

An integral part of current and next generation groundbased astronomical telescopes are laser guide star (LGS) adaptive optics (AO) systems. Many systems will utilize multiple mesospheric sodium LGSs in order to implement laser tomography for ground-layer adaptive optics (GLAO), laser tomography adaptive optics (LTAO), multiobject adaptive optics (MOAO) or multi-conjugate adaptive optics (MCAO) [1-5]. Real-time turbulence profiling is necessary for these wide-field $\mathrm{AO}$ systems to tune their tomographic wavefront reconstruction algorithm $[6,7]$ and perform a variety of image post-processing tasks involving point-spread function reconstruction [8]. This paper describes a computationally efficient and accurate numerical technique inspired by the slope detection and ranging (SLODAR) method [9-13] to perform this task in real-time from properly selected Shack-Hartmann wavefront sensor (WFS) measurements accumulated over a few hundred frames from a pair of LGSs, thus eliminating the need for an additional instrument. A somewhat similar SLODAR-like technique was presented earlier in 2010 [14]. Of course, turbulence sensing with crossed beams has been known for some time [15]. The main conclusions of our analysis are the following:

1. Sensitivity of the method to global tip/tilt and focus can be eliminated by correlating the local curvature of WFS measurements, a feature desirable on account of the uncertain position of LGSs [16] and spatio-temporal fluctuations of the mesospheric sodium layer [17].
2. Turbulence profiling is intrinsically performed at nonequispaced layer altitudes given by

$$
h_{k}=\frac{d_{k}}{\theta+d_{k} / H}
$$

where $k$ denotes layer index $\left(k=0,1, \cdots, n_{l}-1\right), d_{k}=k \Delta$ denotes baseline number $k$ used by the SLODAR-like algorithm, $\Delta$ is the LGS WFS subaperture size, $\theta>0$ is the relative angular separation between the LGS pair (assumed to be either along the $x$ or $y$ direction without loss of generality), and $H$ is the mean altitude of the LGS pair.

3 . Resampling of the turbulence profile estimate computed at altitudes given by Eq. (1) to arbitrary altitudes can be performed if necessary by application of a downsampling matrix computed with triangular [multiaperture scintillation sensor (MASS)-like] interpolating functions. Fine vertical sampling (achieved with a widely separated LGS pair) is, however, desirable for wide-field AO concepts to reduce the requirement for accurate knowledge of layer altitudes [18].

4. Relative estimation errors in the Fried parameter $r_{0}$, the isoplanatic angle $\theta_{0}$, and the generalized dualconjugate isoplanatic angle $\theta_{2}$ [19] are all within a few percent after a few hundred frames of accumulated measurements.

The paper has been structured as follows. Section 2 discusses the proposed SLODAR-like numerical technique, including changes made to the standard method for use 
with LGSs. Section 3 presents a theoretical influence function analysis illustrating the impulse response of the method to high-resolution turbulence profiles. Finally, performance is assessed in Section 4 in the context of the Thirty Meter Telescope (TMT) MCAO system via end-toend wave optics Monte Carlo simulations. Section 5 concludes the study.

\section{SLODAR-LIKE METHOD}

\section{A. Preliminaries}

In what follows, atmospheric turbulence has been discretized into layers at arbitrary altitudes $h_{k}$ [not necessarily given by Eq. (1)] separated by $\Delta_{k}=h_{k+1}-h_{k}$. Each layer represents an optical wavefront, i.e., an optical path difference (OPD) in units of meters $\phi_{k}$, and is fully characterized by its second-order statistics expressed as a structure function, covariance matrix, or power spectral density (PSD), all proportional to the layer strength:

$$
p_{k}=\frac{r_{0, k}^{-5 / 3}}{(2 \pi / \lambda)^{2}}=0.423 \int_{h_{k-1}}^{h_{k+1}} C_{n}^{2}(h) H_{k}(h) d h,
$$

where $r_{0, k}$ denotes the Fried parameter for layer $k$, and $H_{k}(h)$ is a triangular MASS-like interpolating function given by

$$
H_{k}(h)=\left\{\begin{array}{cc}
1-\left|h-h_{k}\right| / \Delta_{k}, & \text { if } h_{k} \leqslant h \leqslant h_{k+1} \\
1-\left|h-h_{k}\right| / \Delta_{k-1}, & \text { if } h_{k-1} \leqslant h \leqslant h_{k} \\
0, & \text { else }
\end{array} .\right.
$$

Note that $p_{k}$ is wavelength independent. Equation (2) can be rewritten in matrix form by discretizing the integral using a quadrature integration rule (e.g., a trapezoidal or Simpson's rule):

$$
\begin{aligned}
\vec{p} & =H \vec{p}^{\prime}, \\
\vec{p}^{\prime} & =W \vec{y},
\end{aligned}
$$

where $H_{k l}=H_{k}\left(h=h_{l}\right), W$ denotes a square diagonal matrix containing the quadrature interpolating weights multiplied by 0.423 , and $\vec{y}$ is a vector of $C_{n}^{2}$ values. Each column of $H$ should have unit $L_{1}$ norm in order to preserve the total turbulence strength $\left(L_{1}\right.$ norm of $\left.\bar{p}^{\prime}\right)$.

It is useful to associate to the strength vector $\vec{p}$ the following four turbulence parameters: the global Fried parameter $r_{0}$, the isoplanatic angle $\theta_{0}$, the generalized isoplanatic angle for a MCAO system with two deformable mirrors (DMs) respectively conjugate to $h=0$ and $h$ $=h_{\mathrm{DM}}, \theta_{2}$, and the Greenwood frequency $f_{\mathrm{G}}$. These parameters can be evaluated from the following expressions:

$$
\begin{aligned}
r_{0}^{-5 / 3} & =\sum_{k} r_{0, k}^{-5 / 3}, \\
\theta_{0}^{-5 / 3} & =\sum_{k} \theta_{0, k}^{-5 / 3}, \\
\frac{\theta_{0, k}^{-5 / 3}}{(2 \pi / \lambda)^{2}} & =\left(\frac{2.914}{0.423}\right) p_{k} h_{k}^{5 / 3},
\end{aligned}
$$

$$
\begin{aligned}
\theta_{2}^{-5 / 3}= & \sum_{k} \theta_{2, k}^{-5 / 3}, \\
\frac{\theta_{2, k}^{-5 / 3}}{(2 \pi / \lambda)^{2}}= & \left(\frac{2.914}{0.423}\right) p_{k} F_{2}\left(h_{k}\right), \\
F_{2}\left(h_{k}\right)= & \frac{h_{k}^{5 / 3}}{2}+\frac{\left|h_{k}-h_{\mathrm{DM}}\right|^{5 / 3}}{2}-\frac{h_{\mathrm{DM}}^{5 / 3}}{4} \\
& -\frac{h_{\mathrm{DM}}^{-5 / 3}}{4}\left(h_{k}^{5 / 3}-\left|h_{k}-h_{\mathrm{DM}}\right|^{5 / 3}\right)^{2}, \\
f_{\mathrm{G}}^{5 / 3}= & \sum_{k} f_{\mathrm{G}, k}^{5 / 3}, \\
\frac{f_{\mathrm{G}, k}^{5 / 3}}{(2 \pi / \lambda)^{2}}= & \left(\frac{0.102}{0.423}\right) p_{k} v_{k}^{5 / 3},
\end{aligned}
$$

where $v_{k}$ denotes wind speed at altitude $h_{k}$. In what follows, all of these turbulence parameters will be quoted at a wavelength $\lambda$ of $500 \mathrm{~nm}$.

The proposed method for real-time estimation of $\vec{p}$ with a pair of LGS WFSs is targeted toward tuning of the tomographic wavefront reconstruction algorithms used by laser tomography AO systems (GLAO,LTAO,MOAO,M$\mathrm{CAO}$ ). These wavefront reconstruction algorithms incorporate the covariance matrix of $\phi_{k}$ (or its inverse, depending on algorithm formulation), which is proportional to $p_{k}$ $[6,7]$.

\section{B. Measurement Description}

The geometry of the SLODAR technique is illustrated in Fig. 1 for a pair of natural guide stars (NGSs) with angular coordinates $\vec{\varphi}=(\varphi, 0)$ and $\vec{\varphi}^{\prime}=\left(\varphi^{\prime}, 0\right)$. The relative angular coordinate is $\vec{\theta}=\left(\theta=\varphi-\varphi^{\prime}, 0\right)$. The fact that $\theta>0$ en-

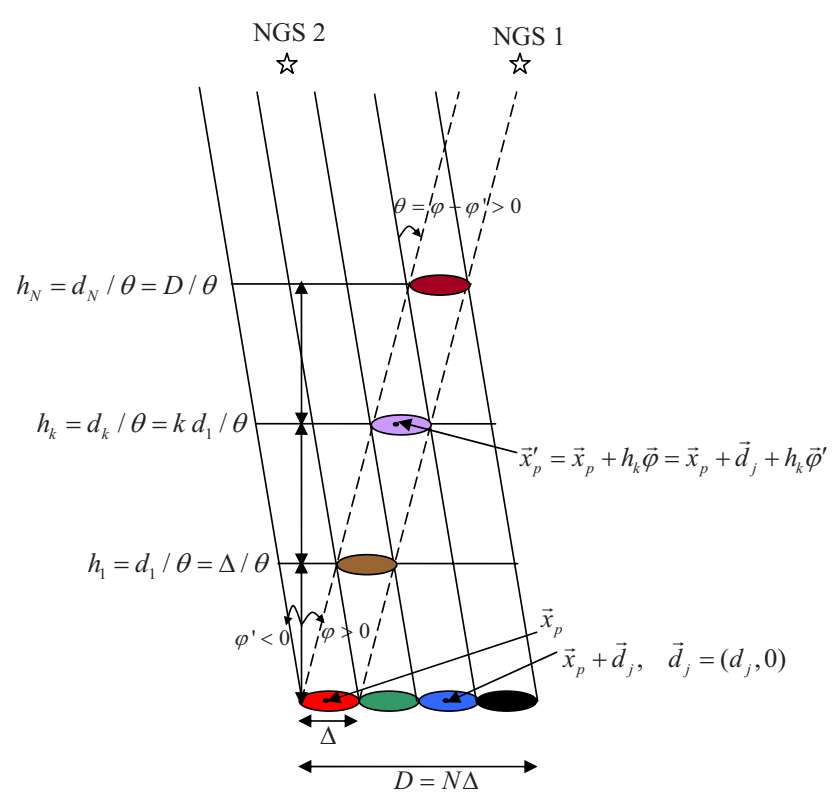

Fig. 1. (Color online) Illustration of the geometry of the SLODAR technique for a pair of NGS Shack-Hartmann WFSs. $\theta$ denotes the relative angular coordinate of the NGS pair and $\Delta$ the NGS WFS subaperture size. 
sures that both beams cross at a positive altitude. For this geometry, turbulence profile estimation is performed at equispaced layer altitudes given by $h_{k}=d_{k} / \theta$, which is the limit case of Eq. (1) when $H \rightarrow \infty$. $\vec{x}_{p}$ denotes a selected subaperture coordinate on the first WFS and $\vec{x}_{p}+\vec{d}_{j}$ a selected coordinate on the second WFS. $\vec{d}_{j}=\left(d_{j}, 0\right)$ is the baseline vector $j$, chosen such that the pair of rays connecting $\vec{x}_{p}$ to the first NGS and $\vec{x}_{p}+\vec{d}_{j}$ to the second NGS intersect at altitude $h_{k}$, which occurs for the choice $\vec{d}_{j}$ $=h_{k} \vec{\theta}$, i.e., when the projected baseline at altitude $h_{k}$ is null: $\vec{d}_{j}^{\prime}=\vec{d}_{j}-h_{k} \vec{\theta}=\overrightarrow{0}$.

The proposed turbulence profile estimation technique follows the same geometry as in Fig. 1 with the only difference being the use of finite range guide stars. The essence of the technique is to fit accumulated measured covariances between properly selected measurements from a pair of LGS WFSs to a correlation model. Note that in closed-loop contexts, LGS WFS measurements need to be converted into "pseudo-open loop" measurements [7], i.e., partially recovered open-loop gradients obtained by adding to the closed-loop measurements the gradients produced by the DM commands (modeled using a geometric interation matrix).

In order to introduce the correlation model, we start by expressing a gradient measurement along the $\alpha$ (either $x$ or $y$ ) direction $s_{\alpha}\left(\vec{x}_{p}\right)$ on a selected square fully illuminated WFS subaperture at coordinate $\vec{x}_{p}$ looking at an angle $\vec{\varphi}=(\varphi, 0)$ as a sum over contributions from each turbulence layer:

$$
s_{\alpha}\left(\vec{x}_{p}\right)=\frac{1}{\Delta^{2}} \sum_{k} \int \operatorname{rec}\left(\frac{\vec{x}-\vec{x}_{p}}{\Delta}\right) \frac{\partial}{\partial \alpha} \phi_{k}\left(\xi_{k} \vec{x}+h_{k} \vec{\varphi}\right) d^{2} \vec{x},
$$

where $\vec{x}$ denotes the aperture-plane coordinates, and $\xi_{k}$ $=1-h_{k} / H<1$ denotes the cone compression factor for a LGS at altitude $H$ and a layer at altitude $h_{k} . s_{\alpha}$ has units of angle (OPD separation over subaperture size). Rewriting Eq. (14) in terms of the aperture-plane coordinates projected onto atmospheric layer $k, \vec{x}^{\prime}=\xi_{k} \vec{x}+h_{k} \vec{\varphi}$, yields

$$
\begin{aligned}
& s_{\alpha}\left(\vec{x}_{p}\right)=\frac{1}{\Delta^{2}} \sum_{k} \int \omega_{k}^{\alpha}\left(\vec{x}^{\prime}\right) \phi_{k}\left(\vec{x}^{\prime}+\vec{x}_{p}^{\prime}\right) d^{2} \vec{x}^{\prime} \\
& \omega_{k}^{\alpha}\left(\vec{x}^{\prime}\right)=\frac{1}{\xi_{k}} \operatorname{rec}\left(\frac{\vec{x}^{\prime}}{\xi_{k} \Delta}\right) \frac{\partial}{\partial \alpha^{\prime}} .
\end{aligned}
$$

Since global tip/tilt and focus are poorly measured by LGS WFSs on account of LGS angular position and range uncertainty $[16,17]$, it is necessary to correlate a local curvature (i.e., second-order difference) of gradients to eliminate sensitivity to these modes. This differencing operation is performed on the measurements from three adjacent subapertures as follows:

$$
m_{\alpha}\left(\vec{x}_{p}\right)=s_{\alpha}\left(\vec{x}_{p}-\vec{\delta}_{\alpha}\right)-2 s_{\alpha}\left(\vec{x}_{p}\right)+s_{\alpha}\left(\vec{x}_{p}+\vec{\delta}_{\alpha}\right),
$$

where $\vec{\delta}_{\alpha}=(\Delta, 0)$ when $\alpha=x$, and $\vec{\delta}_{\alpha}=(0, \Delta)$ when $\alpha=y$. Substituting Eq. (15) into Eq. (17) yields

$$
\begin{aligned}
& m_{\alpha}\left(\vec{x}_{p}\right)=\frac{1}{\Delta^{2}} \sum_{k} \int \mu_{k}^{\alpha}\left(\vec{x}^{\prime}\right) \phi_{k}\left(\vec{x}^{\prime}+\vec{x}_{p}^{\prime}\right) d^{2} \vec{x}^{\prime} \\
& \mu_{k}^{\alpha}\left(\vec{x}^{\prime}\right)=\omega_{k}^{\alpha}\left(\vec{x}^{\prime}-\xi_{k} \vec{\delta}_{\alpha}\right)-2 \omega_{k}^{\alpha}\left(\vec{x}^{\prime}\right)+\omega_{k}^{\alpha}\left(\vec{x}^{\prime}+\xi_{k} \vec{\delta}_{\alpha}\right) .
\end{aligned}
$$

\section{Measurement Covariances}

It is convenient to rewrite Eq. (18) in the Fourier domain:

$$
m_{\alpha}\left(\vec{x}_{p}\right)=\frac{1}{\Delta^{2}} \sum_{k} \int \widehat{\mu_{k}^{\alpha}}\left(\vec{f}^{\prime}\right) \widehat{\phi_{k}}\left(\vec{f}^{\prime}\right) \exp \left(2 i \pi \vec{f}^{\prime \mathrm{T}} \vec{x}_{p}^{\prime}\right),
$$

where the Fourier transformed kernel functions are given by

$$
\begin{aligned}
& \widehat{\mu_{k}^{\alpha}}\left(\vec{f}^{\prime}\right)=2 \widehat{\omega_{k}^{\alpha}}\left(\vec{f}^{\prime}\right)\left[\cos \left(2 \pi \vec{f}^{\prime \mathrm{T}} \xi_{k} \vec{\delta}_{\alpha}\right)-1\right], \\
& \widehat{\omega_{k}^{\alpha}}\left(\vec{f}^{\prime}\right)=-2 i \pi f_{\alpha}^{\prime} \frac{\left(\xi_{k} \Delta\right)^{2}}{\xi_{k}} \operatorname{sinc}\left(f_{x}^{\prime} \xi_{k} \Delta\right) \operatorname{sinc}\left(f_{y}^{\prime} \xi_{k} \Delta\right) .
\end{aligned}
$$

The correlation model between measurement $m_{\alpha}\left(\vec{x}_{p}\right)$ obtained on the first WFS and $m_{\alpha}^{\prime}\left(\vec{x}_{p}+\vec{d}_{j}\right)$ on the second WFS looking at an angle $\vec{\varphi}^{\prime}=\left(\varphi^{\prime}, 0\right)$ with $\theta=\varphi-\varphi^{\prime}>0$ can now be formulated as follows:

$$
\left\langle m_{\alpha}\left(\vec{x}_{p}\right) m_{\alpha}^{\prime}\left(\vec{x}_{p}+\vec{d}_{j}\right)\right\rangle=\sum_{k} A_{j k}^{\alpha} p_{k},
$$

where the matrix element $A_{j k}^{\alpha}$ cross-coupling baseline $j$ to layer $k$ is given by

$$
A_{j k}^{\alpha}=\int d^{2} \vec{f}^{\prime}\left|\widehat{\mu_{k}^{\alpha}}\left(\vec{f}^{\prime}\right)\right|^{2} \operatorname{PSD}_{0}\left(\vec{f}^{\prime}\right) \exp \left(2 i \pi \vec{f}^{\prime} \mathrm{T} \vec{d}_{j}^{\prime}\right),
$$

$$
\operatorname{PSD}_{0}\left(\vec{f}^{\prime}\right)=2.29 \times 10^{-2}\left(\left\|\vec{f}^{\prime}\right\|^{2}+L_{0}^{-2}\right)^{-11 / 6}
$$

where $\vec{d}_{j}^{\prime}=\xi_{k} \vec{d}_{j}-h_{k} \vec{\theta}$. In other words, $A_{j k}^{\alpha}$, is expressed as an inverse Fourier transform of a filtered unit strength von Kármán PSD evaluated at the projected baseline coordinate $\vec{d}_{j}^{\prime}$. Since $A$ is a small matrix, each matrix element (24) can be numerically evaluated as a double summation of the discretized integrand. As illustrated in Fig. 1 , when the relative LGS angular coordinate is either along the $x$ or $y$ direction (a requirement that can be incorporated into the opto-mechanical design of the system by maintaining a fixed LGS asterism orientation in the LGS WFS coordinate system), a natural choice of layer altitudes $h_{k}$ at which turbulence strength will be estimated is that given in Eq. (1). For this choice of altitudes, we have

$$
d_{j}^{\prime}=\left[j-k\left(\frac{1+j \Delta /(\theta H)}{1+k \Delta /(\theta H)}\right)\right] \Delta .
$$

Note that Eqs. (24) and (26) indicate that $A^{\alpha}$ is nonsymmetric for finite range guide stars $(H<\infty)$ and becomes symmetric in the limit $H \rightarrow \infty$. Note also that measurement noise is uncorrelated between distinct WFSs, and hence measurement noise statistics does not appear in Eq. (24). 


\section{Turbulence Profile Estimation}

Finally, Eq. (23) leads to a matrix system of the form $A^{\alpha} \vec{p}=\vec{b}^{\alpha}$, where $\vec{b}^{\alpha}$ denotes the vector of measured correlations with the $j$ th component equal to

$$
\left[\vec{b}^{\alpha}\right]_{j}=\left(\frac{1}{n_{p}}\right) \sum_{p=1}^{n_{p}}\left\langle m_{\alpha}\left(\vec{x}_{p}\right) m_{\alpha}^{\prime}\left(\vec{x}_{p}+\vec{d}_{j}\right)\right\rangle,
$$

where $n_{p}$ denotes the total number of valid subapertures over which the correlations are averaged. The size of $A^{\alpha}$ is thus equal to $n_{d} \times n_{l}$, where $n_{d}$ denotes the number of baseline vectors $\vec{d}_{j}$ used and $n_{l}$ the number of reconstructed layers. Note that $n_{d}=n_{l}$ when altitudes are selected as in Eq. (1). Concatenating the above $\alpha=x$ and $\alpha$ $=y$ matrix systems leads to the augmented system $A \vec{p}=\vec{b}$, where $A$ has size $2 n_{d} \times n_{l}$. The solution $\hat{\vec{p}}$ of this overdetermined system is given by the least-squares projection of $\vec{b}$ onto the range of $A$ :

$$
\begin{aligned}
\hat{\vec{p}} & =\underset{\vec{p}}{\arg \min }\|A \vec{p}-\vec{b}\|^{2}=A^{\dagger} \vec{b}, \\
A^{\dagger} & =\left(A^{\mathrm{T}} A\right)^{-1} A^{\mathrm{T}} .
\end{aligned}
$$

Note that Eq. (28) does not guarantee non-negativity of the solution vector. When negative entries occur, these components are set to zero, and the minimization is repeated for the reduced-size system obtained by removing the corresponding columns of $A$.

\section{INFLUENCE FUNCTION ANALYSIS}

In order to analyze the response of the above discrete estimator $A^{\dagger}$ to high-resolution turbulence profiles, we first compute $A^{\dagger}$ for a small number $n_{l}$ of altitudes selected as in Eq. (1) using $n_{d}=n_{l}$ baselines $d_{j}=j \Delta$ with $j=0, \cdots, n_{l}$ -1 . A down-sampling matrix can then be formed by postmultiplication of $A^{\dagger}$ with a high-resolution kernel $B$ computed for a much larger number of turbulence altitudes, but with the same baselines $d_{j}$ used to build the lower resolution kernel $A$. We will call influence functions the columns of the down-sampling matrix $Q=A^{\dagger} B$, since they quantify how a given input unit strength layer on a finely sampled altitude grid is estimated by $A^{\dagger}$ on a coarser grid.

This procedure has been carried out for $A^{\dagger}$ estimating turbulence on either $n_{l}=6$ or $n_{l}=12$ layers at altitudes selected as in Eq. (1) with $\Delta=0.5 \mathrm{~m}$ and $\theta=35^{\prime \prime}$ for the 6 -layer case and $\theta=67^{\prime \prime}$ for the 12-layer case, and for $B$ computed for 27 layers. For the 12-layer case, $Q$ has been pre-multiplied by $H$ as given in Eq. (3) to form a 6-layer estimate. The chosen values of $\theta$ correspond to two possible LGS pairs from the TMT 70" diameter 6-LGS asterism (pentagon+on-axis) [1]. The resulting 27 influence functions are displayed in Fig. 2 for the $\theta=35^{\prime \prime}$ case, and in Fig. 3 for the $\theta=67$ " case. It is seen that each influence function has non-zero weights at the selected 6 layer altitudes, and that both narrow and wide LGS separations produce similar influence functions. Slightly negative weights are also observed in the tails of these influence functions, a feature (probably introduced by discretization effects) that is unphysical since each $p_{k}$ should be nonnegative.
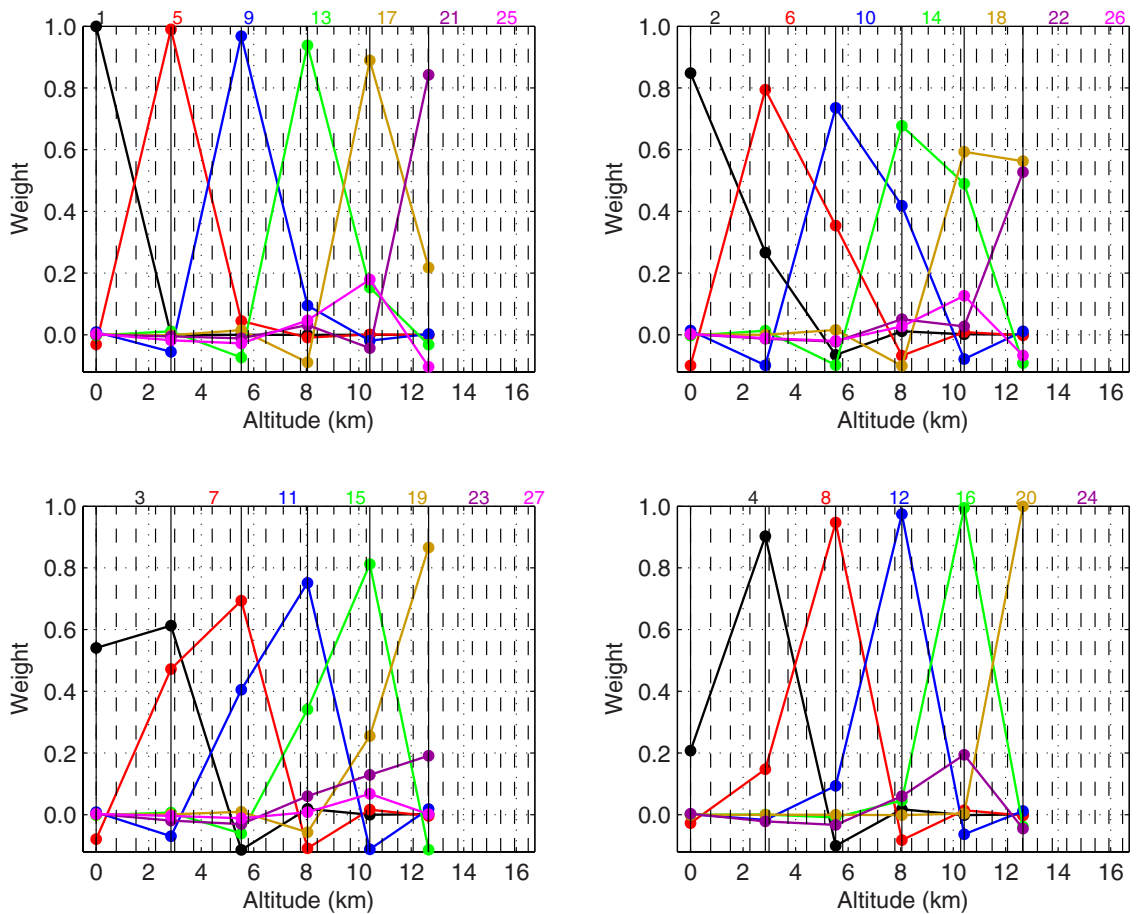

Fig. 2. (Color online) Sample influence functions (columns of $Q=A^{\dagger} B$ ) illustrating how a 27-layer turbulence profile is sensed at six altitudes selected as in Eq. (1) with $\Delta=0.5 \mathrm{~m}$ and $\theta=35^{\prime \prime}$. The 27 influence functions have been grouped into four panels. The numbers at the top of each panel indicate which influence is plotted and the location of the corresponding layer, and they provide a color-coded pointer to the corresponding influence function. 

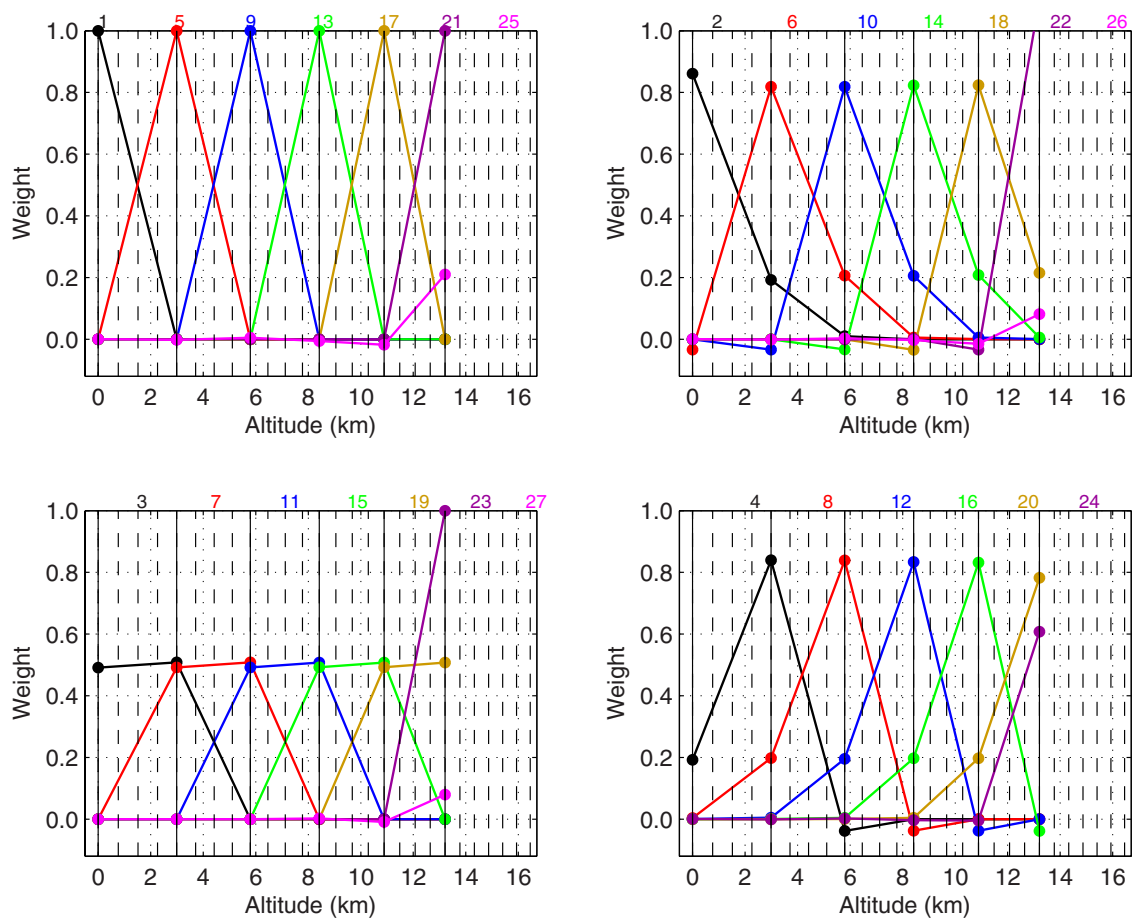

Fig. 3. (Color online) Same as in Fig. 2 but for $A^{\dagger}$ estimating turbulence at 12 altitudes selected as in Eq. (1) with $\Delta=0.5 \mathrm{~m}$ and $\theta=67 "$, followed by down-sampling to six layers via Eq. (4).

\section{SAMPLE SIMULATION RESULTS}

Detailed Monte Carlo simulations were conducted in order to test the accuracy of the proposed method in the context of the TMT MCAO system [1], which is an order 60 $\times 60$ wavefront sensing and order $63 \times 63$ correction on the ground conjugated deformable mirror (DM) and order $76 \times 76$ correction on the $h_{\mathrm{DM}}=11.2 \mathrm{~km}$ conjugated DM. Our study has been performed for high-resolution simulated turbulence profiles obtained from generalized scintillation detection and ranging (SCIDAR) measurements over Mauna Kea during four nights in 2002 [20]. Two sample profiles corresponding to a median $r_{0}$ are displayed in Fig. 4 as dimensionless relative weights $\omega_{k}$ $=p_{k} / \Sigma_{k} p_{k}$. The $N=65$-layer profile was directly obtained from generalized SCIDAR measurements, whereas the $N=27$-layer profile was obtained from the former by application of the down-sampling matrix given in Eq. (3). Both profiles are characterized by $r_{0}=0.18 \mathrm{~m}, \theta_{0}=2.8^{\prime \prime}$, and $\theta_{2}\left(h_{\mathrm{DM}}=11.2 \mathrm{~km}\right)=10.9^{\prime \prime}$ at $\lambda=500 \mathrm{~nm}$. Highresolution night-time wind profiles were obtained from the NCEP/NCAR (National Center for Environmental Prediction/Atmospheric Research) reanalysis project [21]. The Greenwood frequency associated with the two profiles displayed in Fig. 4 is $f_{\mathrm{G}}=22 \mathrm{~Hz}$ at $\lambda=500 \mathrm{~nm}$.

Both the 65-layer and 27-layer turbulence profiles displayed in Fig. 4 were simulated. Phase screens were generated via fast Fourier transforms (FFTs) on $4096 \times 4096$ grids sampled at $1 / 64 \mathrm{~m}$ with $L_{0}=30 \mathrm{~m}$ outer scale. All simulations included the effects of LGS WFS measurement noise and LGS WFS nonlinearity resulting from the use of $a$ constrained matched filter centroiding algorithm [22].

The TMT MCAO tomographic wavefront reconstruction algorithm is required to reconstruct $n_{l}=6$ turbulence lay- ers [7]. Tuning of the weight vector $\vec{p}$ of these layers was achieved by periodic update of this vector every 100 frames, starting 400 frames after the LGS loop running at $800 \mathrm{~Hz}$ had been closed. The update was provided by the previously described SLODAR-like algorithm. An intentional mistuning of $\vec{p}$ used by the tomography algorithm at the beginning of the simulation was performed in order to provide insight into the required number of frames to accumulate the SLODAR covariances to yield optimal closed-loop performance.

Figure 5 displays results for the simulated 27-layer atmosphere and for the SLODAR algorithm using the nar-

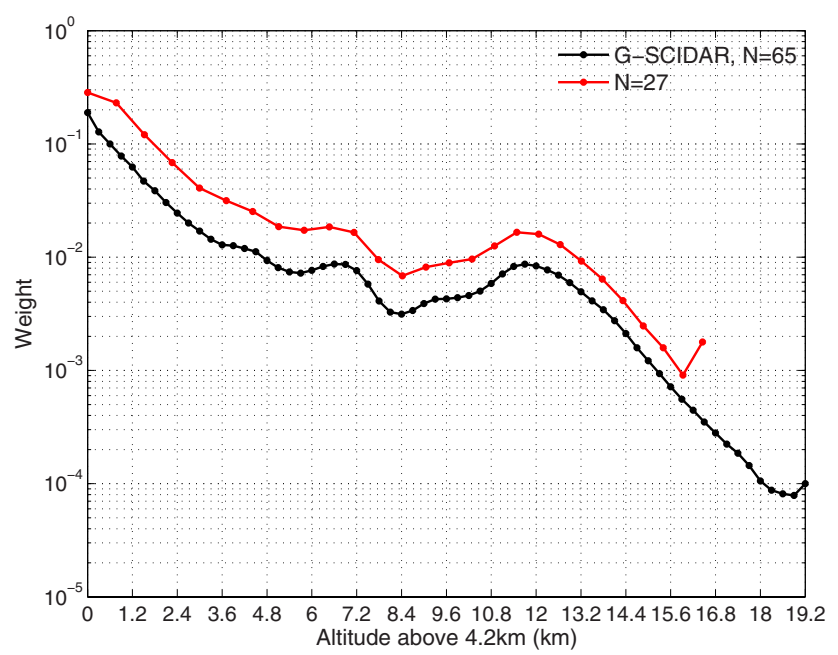

Fig. 4. (Color online) Median seeing Mauna Kea atmospheric turbulence profiles obtained from [20]. The 65-layer profile was obtained from generalized SCIDAR, while the 27-layer one was generated by application of the MASS-like down-sampling matrix Eq. (4) to the former profile. 

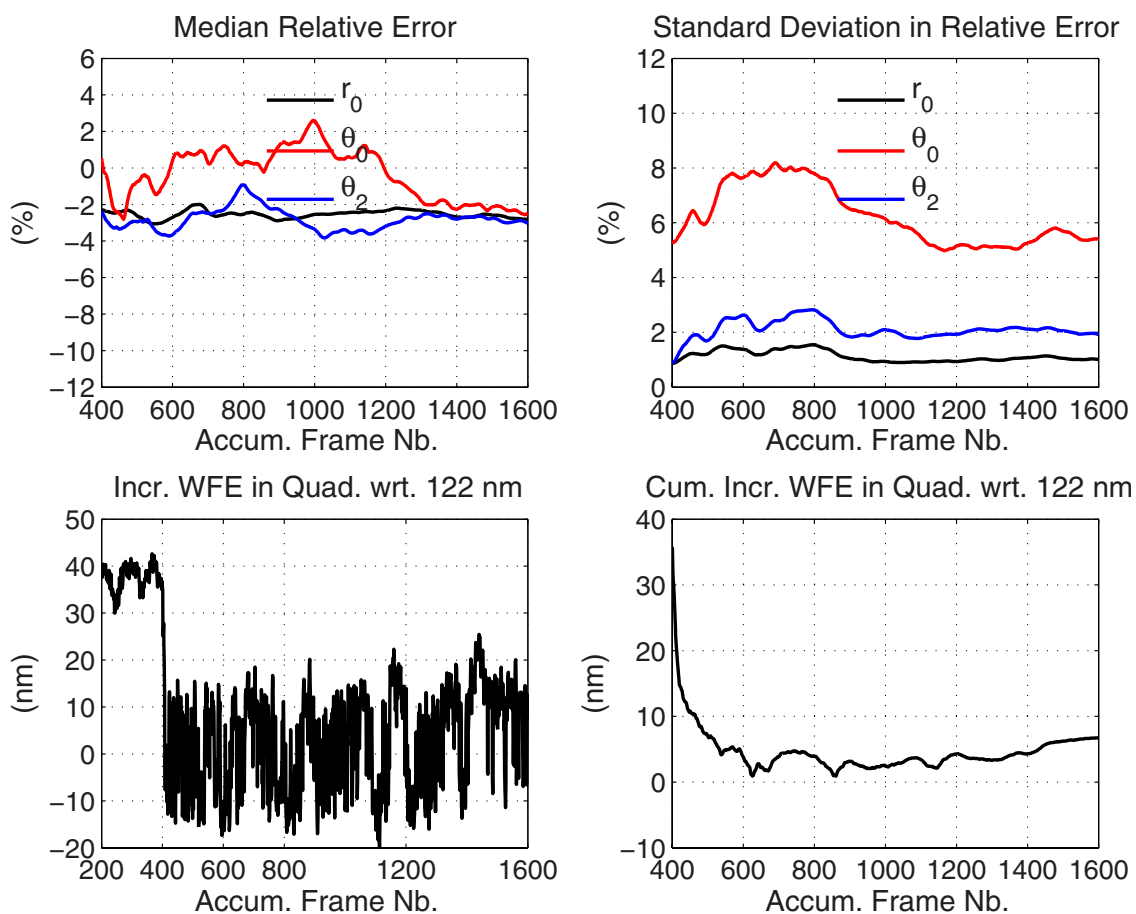

Fig. 5. (Color online) Results for the simulated 27-layer atmosphere and for the SLODAR-like algorithm using the narrow LGS pair separated by $\theta=35^{\prime \prime}$. Upper panels illustrate the time history of the median and standard deviation of the relative estimation error in $r_{0}$, $\theta_{0}$, and $\theta_{2}$ over five different normally distributed realizations of the atmosphere. Bottom panels illustrate the time history of the residual WFE and of its cumulative temporal average delivered by the TMT MCAO system and plotted as incremental WFE in quadrature with respect to a performance estimate of $122 \mathrm{~nm}$ RMS obtained in simulations where the reconstruction algorithm is well tuned to the turbulence profile. Positive values indicate degraded performance (higher WFE) compared to $122 \mathrm{~nm}$, while negative values indicate improved performance (lower WFE).

row LGS pair separated by $\theta=35^{\prime \prime}$. Upper panels illustrate the time history of the median and standard deviation of the relative estimation error in $r_{0}, \theta_{0}$, and $\theta_{2}$ over five different normally distributed realizations of the atmosphere. It is seen that all of these errors are within a

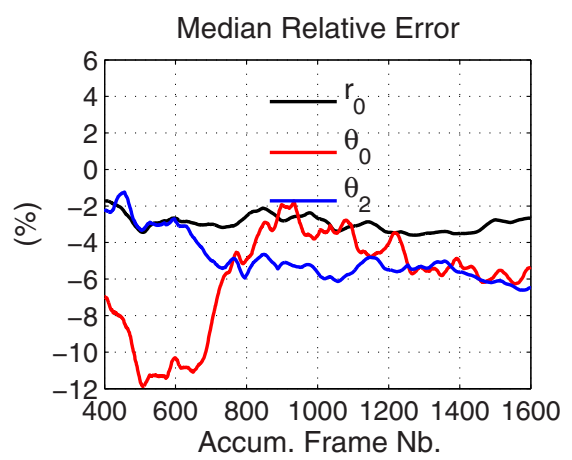

Incr. WFE in Quad. wrt. $122 \mathrm{~nm}$

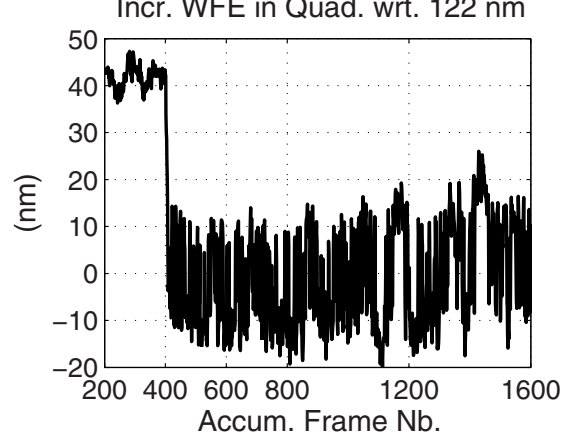

few percent. Bottom panels illustrate the time history of the residual wavefront error (WFE) and of its cumulative temporal average delivered by the MCAO system and plotted as incremental WFE in quadrature with respect to a performance estimate of $122 \mathrm{~nm}$ root-mean-square

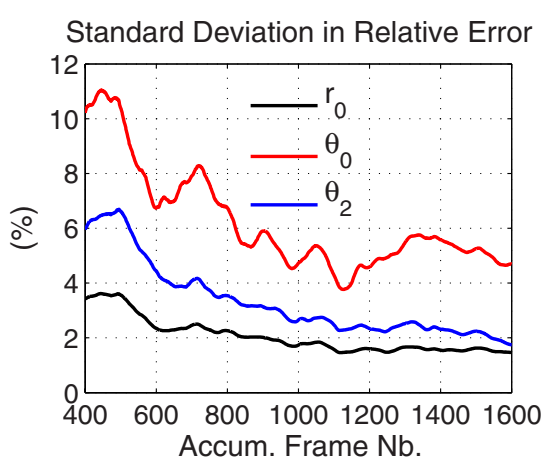

Cum. Incr. WFE in Quad. wrt. $122 \mathrm{~nm}$

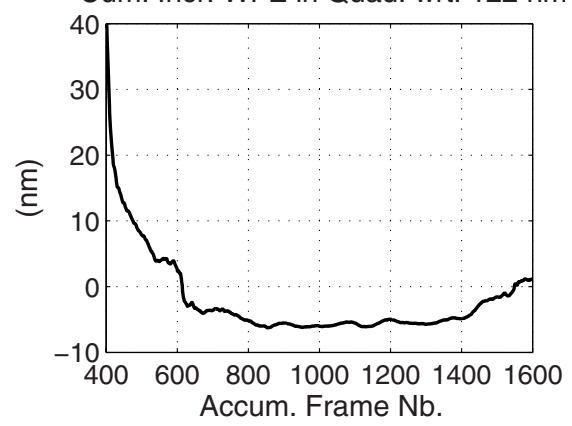

Fig. 6. (Color online) Same as in Fig. 5 but with the SLODAR-like algorithm employing the wider LGS pair separated by $\theta=67 "$. 

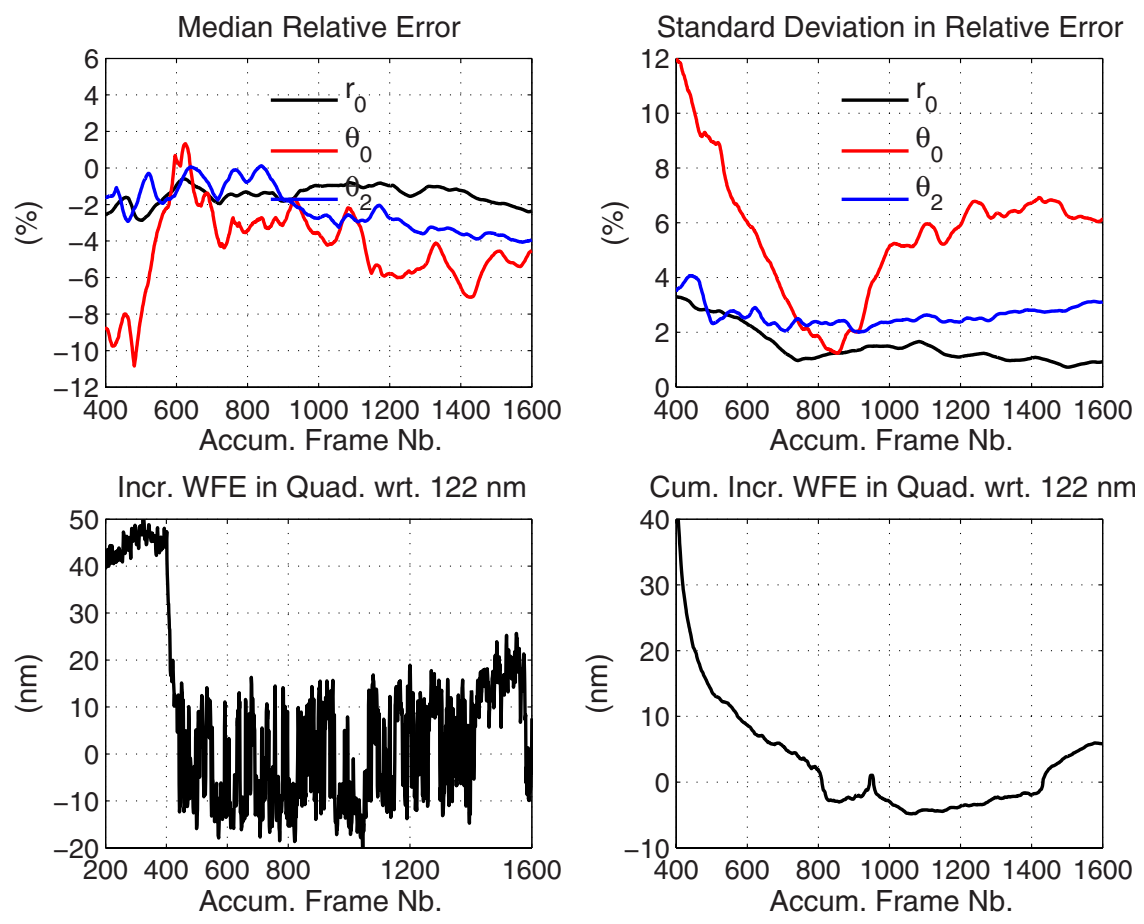

Fig. 7. (Color online) Same as in Fig. 6 but for the simulated 65-layer atmosphere.

(RMS) obtained in simulations where the reconstruction algorithm is well tuned to the turbulence profile. Positive values indicate degraded performance (higher WFE) compared to $122 \mathrm{~nm}$, while negative values indicate improved performance (lower WFE). Note that this performance estimate does not include implementation errors and tip/tilt and tilt anisoplanatism errors [23]. It is seen that only a few hundred accumulation frames are needed for optimal closed-loop residual WFE. Figure 6 illustrates results for the same scenario as in Fig. 5 but with the SLODAR algorithm employing the wider LGS pair separated by $\theta$ $=67 "$. Results are similar to those obtained for the narrower LGS pair with the caveat that a longer accumulation time may be required on account of the wider baselines used in this case. Finally, Fig. 7 illustrates results for the same scenario as in Fig. 6 but for the simulated 65-layer atmosphere. Results are also very similar to those obtained for the simulated 27-layer atmosphere.

\section{CONCLUSIONS}

We have described a computationally efficient and accurate numerical technique requiring a short averaging time inspired by the SLODAR method to tune tomographic wavefront reconstruction algorithms for wide-field adaptive optics systems on large to extremely large telescopes, and to perform a variety of image post-processing tasks involving point-spread function reconstruction. We have shown that this task can be performed in real time from properly selected Shack-Hartmann WFS measurements from a pair of LGSs, eliminating the need for an additional instrument.

\section{ACKNOWLEDGMENTS}

The authors gratefully acknowledge the support of the TMT partner institutions. They are the Association of $\mathrm{Ca}$ - nadian Universities for Research in Astronomy (ACURA), the California Institute of Technology, and the University of California. This work was supported as well by the Gordon and Betty Moore Foundation, the Canada Foundation for Innovation, the Ontario Ministry of Research and Innovation, the National Research Council of Canada, the Natural Sciences and Engineering Research Council of Canada, the British Columbia Knowledge Development Fund, the Association of Universities for Research in Astronomy (AURA), and the U.S. National Science Foundation.

\section{REFERENCES}

1. B. Ellerbroek, S. Adkins, D. Andersen, J. Atwood, S. Browne, C. Boyer, P. Byrnes, K. Caputa, R. Conan, R. Cousty, D. Erikson, J. Fitzsimmons, F. Gamache, L. Gilles, G. Herriot, P. Hickson, O. Lardiere, P. Morin, J. Pazder, T. Pfrommer, D. Quinn, V. Reshetov, S. Roberts, J.-C. Sinquin, M. Schoeck, M. Smith, G. Tyler, J. Vaughn, J.-P. Véran, C. Vogel, L. Wang, and I. Wevers, "First light adaptive optics systems and components for the Thirty Meter Telescope," Proc. SPIE 7736, 773604-1-773604-14 (2010).

2. P. Hinz, A. Bouchez, M. Johns, S. Shectman, M. Hart, B. McLeod, and P. McGregor, "The GMT adaptive optics system," Proc. SPIE 7736, 77360C-1-77360C-12 (2010).

3. T. Fusco, Y. Cl'enet, S. Meimon, M. Cohen, J. Paufique, C. Petit, D. Gratadour, V. Michau, J.-P. Amans, J.-L. Dournaux, P. Jagourel, H. Schnetler, J.-M. Conan, C. Robert, E. Gendron, G. Rousset, and N. Hubin, "ATLAS: the laser tomographic adaptive optics module for the E-ELT," in Proceedings of 1st AO4ELT Conference-Adaptive Optics for Extremely Large Telescopes (EDP Sciences, 2010), pp. 02002-1-02002-6, available online at ao4elt.edpsciences.org.

4. E. Diolaiti, J.-M. Conan, I. Foppiani, M. Lombini, E. Marchetti, C. Petit, C. Robert, L. Schreiber, A. Baruffolo, M. Bellazzini, G. Bregoli, P. Ciliegi, G. Cosentino, V. Biliotti, S. D'Odorico, T. Fusco, N. Hubin, S. Meimon, and J.-F. Sauvage, "Towards the phase A review of MAORY, the multiconjugate adaptive optics module for the E-ELT," in Pro- 
ceedings of 1st AO4ELT Conference-Adaptive Optics for Extremely Large Telescopes (EDP Sciences, 2010), pp. 02007-1-02007-5, available online at ao4elt.edpsciences.org.

5. G. Rousset, T. Fusco, F. Assemat, T. Morris, E. Gendron, R. Myers, M. Brangier, M. Cohen, N. Dipper, C. Evans, D. Gratadour, P. Jagourel, P. Laporte, D. Le Mignant, M. Puech, C. Robert, H. Schnetler, W. Taylor, F. Vidal, J.-G. Cuby, M. Lehnert, S. Morris, and P. Parr-Burmann, "EAGLE multi-object AO concept study for the E-ELT," in Proceedings of 1st AO4ELT Conference-Adaptive Optics for Extremely Large Telescopes (EDP Sciences, 2010), pp. 02008-1-02008-6, available online at ao4elt.edpsciences.org.

6. T. Fusco, J.-M. Conan, G. Rousset, L. M. Mugnier, and V. Michau, "Optimal wavefront reconstruction strategies for multiconjugate adaptive optics," J. Opt. Soc. Am. A 18, 2527-2538 (2001).

7. L. Gilles and B. L. Ellerbroek, "Split atmospheric tomography using laser and natural guide stars," J. Opt. Soc. Am. A 25, 2427-2435 (2008).

8. M. C. Britton, "The anisoplanatic point-spread function in adaptive optics," Publ. Astron. Soc. Pac. 118, 885-900 (2006).

9. R. W. Wilson, "Slodar: measuring optical turbulence altitude with a Shack-Hartmann wavefront sensor," Mon. Not. R. Astron. Soc. 337, 103-108 (2002).

10. T. Butterley, R. W. Wilson, and M. Sarazin, "Determination of the profile of atmospheric optical turbulence strength from SLODAR data," Mon. Not. R. Astron. Soc. 369, 835845 (2006).

11. N. Védrenne, V. Michau, C. Robert, and J.-M. Conan, " $C_{n}^{2}$ profile measurement from Shack-Hartmann data," Opt. Lett. 32, 2659-2661 (2007).

12. L. Wang, M. Schöck, and G. Chanan, "Atmospheric turbulence profiling with SLODAR using multiple adaptive optics wavefront sensors," Appl. Opt. 47, 1880-1892 (2008).

13. M. Goodwin, C. Jenkins, and A. Lambert, "Improved detection of atmospheric turbulence with SLODAR," Opt. Express 15, 14845-14860 (2007).

14. F. Vidal, E. Gendron, M. Brangier, A. Sevin, G. Rousset, and Z. Hubert, "Tomography reconstruction using the Learn and Apply algorithm," in Proceedings of 1st AO4ELT Conference-Adaptive Optics for Extremely Large Telescopes (EDP Sciences, 2010), pp. 07001-1-07001-6, available online at ao4elt.edpsciences.org.

15. A. Tokovinin and E. Viard, "Limiting precision of tomographic phase estimation," J. Opt. Soc. Am. A 18, 873-882 (2001).

16. F. Rigaut and E. Gendron, "Laser guide star in adaptive optics: the tilt determination problem," Astron. Astrophys. 262, 677-684 (1992).

17. T. Pfrommer, P. Hickson, and C. Y. She, "A large-aperture sodium fluorescence lidar with very high resolution for mesopause dynamics and adaptive optics studies," Geophys. Res. Lett. 36, L15831 (2009).

18. B. Neichel, T. Fusco, and J.-M. Conan, "Tomographic reconstruction for wide-field adaptive optics systems: Fourier domain analysis and fundamental limitations," J. Opt. Soc. Am. A 26, 219-235 (2009).

19. A. Tokovinin, M. Le Louarn, and M. Sarazin, "Isoplanatism in multiconjugate adaptive optics system," J. Opt. Soc. Am. A 17, 1819-1827 (2000).

20. A. Tokovinin, J. Vernin, A. Ziad, and M. Chun, "Optical turbulence profiles at Mauna Kea measured by MASS and SCIDAR," Publ. Astron. Soc. Pac. 117, 395-400 (2005).

21. E. Kalnay, M. Kanamitsu, R. Kistler, W. Collins, D. Deaven, L. Gandin, M. Iredell, S. Saha, G. White, J. Woollen, Y. Zhu, A. Leetmaa, R. Reynolds, M. Chelliah, W. Ebisuzaki, W. Higgins, J. Janowiak, K. C. Mo, C. Ropelewski, J. Wang, R. Jenne, and D. Joseph, "The NCEP/NCAR 40-year reanalysis project,” Bull. Am. Astron. Soc. 77, 437470 (1996).

22. L. Gilles and B. L. Ellerbroek, "Constrained matched filtering for extended dynamic range and improved noise rejection for Shack-Hartmann wavefront sensing," Opt. Lett. 33, 1159-1161 (2008).

23. L. Gilles, L. Wang, and B. Ellerbroek, "Modeling update for the Thirty Meter Telescope laser guide star dual-conjugate adaptive optics system," Proc. SPIE 7036, 77360W-177360W-11 (2010). 